\title{
EGIS 1.0: QR Code Screening Mechanism to Prevent Airline Luggage Loss
}

\author{
Satya Kumar Lalam \\ GITAM University \\ Dept. of Information \\ Technology \\ Visakhapatnam, India-530045
}

\author{
Monica Pragada \\ GITAM University \\ Dept. of Information \\ Technology \\ Visakhapatnam, India-530045
}

\author{
Sudheer Palempati \\ Vignan's Institute of Information \\ Technology \\ Dept. of EEE \\ Visakhapatnam, India-530049
}

\begin{abstract}
Airport check-in uses service counters at airports handling commercial air travel. The check-in is normally handled by an airline itself by a handling agent working on behalf of an airline. Passengers usually hand over any baggage that they do not wish or are not allowed to carry-on to the aircraft's cabin and receive a boarding pass before they can proceed to board their aircraft. The airline check-in's main function, however, is to accept luggage that is to go in the aircraft's cargo. The arriving passengers claim checked-in baggage after disembarking from an airline flight at Baggage Claim Carousels and the airline agents does the unreliable handmade inspection of luggage tags, which inevitably cause that losing and misdeeming luggage. In this paper we propose a QR Code Screening Mechanism which is based on linking and data restoration functionality of QR Code to enhance the reliability.
\end{abstract}

\section{General Terms}

Lost luggage, Baggage claim, Misdirected luggage, Baggage Handling System, Applications of QR Code.

\section{Keywords}

QR Code, Luggage Inspection System, Baggage carousel, Baggage claim, EGIS 1.0, Lost luggage, Baggage claim, Misdirected luggage, Baggage Handling System, Applications of QR Code.

\section{INTRODUCTION}

The most universal process of aviation luggage consign at present is that the passenger should get the boarding card and luggage tag before getting on the plane, then air staff paste the luggage tag on the luggage and boarding card, then consign the luggage. When arriving, the passenger will take back the luggage at luggage claim, and get out of the airdrome after compare the tags between the luggage and the boarding card by air staff. There is some shortages in the process as follows:

(a) High rate of misdeeming. It is difficult to avoid misdeeming when the passengers pick their luggage, for the luggage are so similar or for other reasons.

(b) Low efficiency. By using luggage tag to inspection the luggage, we should need many air staff to inspection. Because the whole process is man-made, it wastes time and result in low efficiency.

(c) Unreliability. It is difficult to avoid man-made error during the process. This manual inspection not only lead inconvenience to the passengers, but also bring the credit and economy impairment of the airport.

According to report released by Office of Aviation Enforcement and Proceedings, U.S. Department of Transportation[6] .Airline industry loses up to 200,000 passenger luggages a year, and lost rate runs to one in 1000 for domestic operations and about 3.30 per 1000 for international. The same thing occurs from time to time around the world every day. The main reason is that passengers confuse luggage at luggage claim since many luggage are similar in appearance when they are picking up their luggage. In addition, with some airport staff's careless inspection, therefore these lug gages will be wrong taken sometimes. Luggage disappearing not only gives passengers annoyance and pressure, but also brings the airways many troubles.

Though some international airports have adopted or plan to adopt RFID system for transporting and security inspection of luggage to improve the accuracy, the RFID label is more expensive than the barcode. The cost of the barcode is the paper and printing ink, which is very cheap, while the price of the radio-label with memory chip is above 2 dollar and the cost of the passive radio-label is above 1 dollar. Furthermore, the cost of basic infrastructure for the RFID system will be much more expensive than that of RFID's label. Besides, the average accuracy of RFID recognition rate is below $90 \%$, so it is difficult to adopt RFID technology for most airline companies. After analysis of the shortage of manual inspection manner and RFID technology during luggage inspection, this paper proposes a new luggage inspection system based on QR code. By the link structure of QR code, it can reduce the lost rate of passengers' luggage, and improve the efficiency of the airport. Meanwhile, it can also expand the application field of the two dimension barcode.

\section{BASIC THEORY OF QR CODE}

QR Code[1] is a two-dimensional symbol. It was invented in 1994 by Denso, one of major Toyota group companies, and approved as an ISO international standard (ISO/IEC18004) in June 2000. This two-dimensional symbol was initially intended for use in production control of automotive parts, but it has become widespread in other fields.

\subsection{The QR Code Structure}

QR Code is a matrix type symbol with a cell structure arranged in a square. It consists of the functionality patterns for making reading easy and the data area where the data is stored. QR Code has finder patterns, alignment patterns, timing patterns, and a quiet zone 


\subsubsection{Finder Pattern}

A pattern for detecting the position of the QR Code. By arranging this pattern at the three corners of a symbol, the position, the size, and the angle of the symbol can be detected. This finder pattern consists of a structure which can be detected in all directions $\left(360^{\circ}\right)$.

\subsubsection{Alignment Pattern}

A pattern for correcting the distortion of the QR Code. It is highly effective for correcting nonlinear distortions. The central coordinate of the alignment pattern will be identified to correct the distortion of the symbol. For this purpose, a black isolated cell is placed in the alignment pattern to make it easier to detect the central coordinate of the alignment pattern.

\subsubsection{Timing Pattern}

A pattern for identifying the central coordinate of each cell in the QR Code with black and white patterns arranged alternately. It is used for correcting the central coordinate of the data cell when the symbol is distorted or when there is an error for the cell pitch. It is arranged in both vertical and horizontal directions

\subsubsection{Quiet Zone}

This is the margin space necessary for reading the QR Code. This quiet zone makes it easier to have the symbol detected from among the image read by the CCD sensor. Four or more cells are necessary for the quiet zone.

\subsubsection{Data Area}

The QR Code data will be stored (encoded) into the data area. The grey part in Figure 1 represents the data area. The data will be encoded into the binary numbers of ' 0 ' and ' 1 ' based on the encoding rule. The binary numbers of ' 0 ' and ' 1 ' will be converted into black and white cells and then will be arranged. The data area will have Reed-Solomon codes incorporated for the stored data and the error correction functionality.

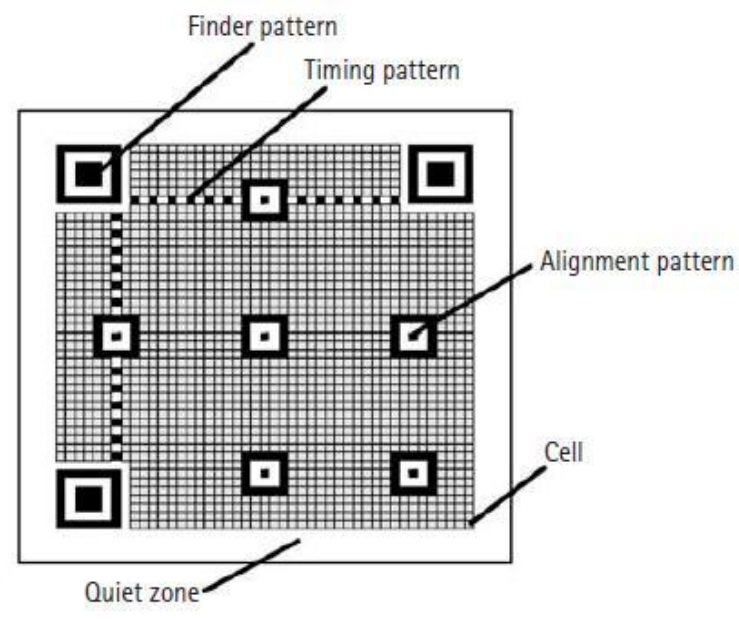

Figure 1: The QR Code Structure

\section{CHARACTERISTICS OF QR CODE}

\subsection{All-Direction $\left(360^{\circ}\right)$ High-Speed Reading}

Please Reading matrix symbols will be implemented by using a CCD sensor (area sensor). The data of the scan line captured by the sensor will be stored into the memory. Then, by using the software, the details will be analyzed, finder patterns identified, and the position/size/angle of the symbol detected, and the decoding process will be implemented. Traditional two-dimensional symbols used to take much time for detecting the position/angle/size of the symbol, and had a problem that their readings were less accurate when compared with those of linear symbols. QR Code has finder patterns for notifying the position of the symbol arranged in three of its corners to enable high-speed reading in all directions $\left(360^{\circ}\right)$. The ratio between black and white among the scan line that runs through the finder patterns is always $1: 1: 3: 1: 1$ when seen from any direction among the $360^{\circ}$ surrounding it. By detecting this specific ratio, the finder pattern can be detected from among the image captured by the CCD sensor to identify the position of the QR Code in a short period of time. Additionally, by identifying the positional relationships of the three finder patterns from among the image field of the CCD sensor, the size (L), the angle (!), and the outer shape of the symbol can be simultaneously detected. By arranging the finder patterns into the three corners of the symbol, the decoding speed of the QR Code can be made 20 times faster than that of other matrix symbols. Additionally, detecting finder patterns can be easily implemented by the hardware, and can also be accelerated.

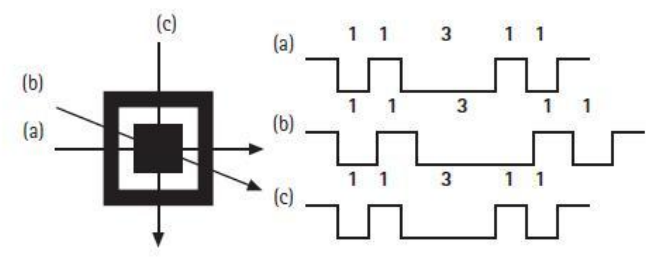

Figure 2: Finder Patterns

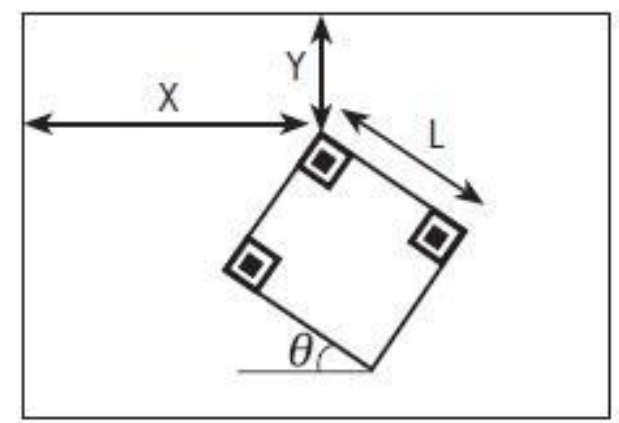

Figure 3: Identifying a QR Code

\subsection{Resistant to Distorted Symbols}

Symbols often get distorted when attached onto a curved surface or by the reader being tilted (angled between the CCD sensor face and the symbol face). To correct this distortion, QR Code has alignment patterns arranged with a regular interval within the range of the symbol. The variance between the Centre position of the alignment pattern estimated from the outer shape of the symbol and the actual Centre position of the alignment pattern will be calculated to have the mappings (for identifying the Centre position of each cell) corrected. This will make the distorted linear/non-linear symbols readable. 


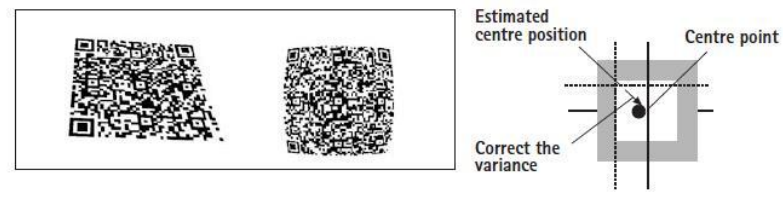

Figure 4: Correcting Distorted Symbols

\subsection{Data Restoration Functionality (Resistant to Smudged or Damaged Symbols)}

QR Code has four different error correction levels $(7 \%, 15 \%$, $25 \%$, and $30 \%$ per symbol area). The error correction functionality is implemented according to each of the smudge/damage, and is utilizing Reed-Solomon code which is highly resistant to burst errors. Reed-Solomon codes are arranged in the QR Code data area. By this error correction functionality, the codes can be read correctly even when they are smudged or damaged up until the error correction level. The error correction level can be configured by the user when he/she creates the symbol. So if the code is highly likely to get smudged in the users' usage environment, it is recommended to have $30 \%$ set for this correction level.
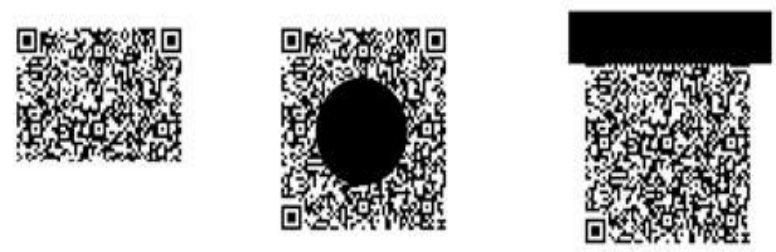

Figure 5: Smudged/Damaged Symbols

\subsection{Linking Functionality of the Symbols}

QR Code has a linking functionality which will enable a single symbol to be represented in several symbols by dividing it. A single symbol can be divided into 16 symbols at maximum. The example shown in Figure 7 is one where a single QR Code is divided into four symbols, and each symbol has an indicator showing how many symbols the original symbol had been divided into and in which order that specific symbol would be among all divided ones. This will enable the entire data to be edited and submitted to the computer regardless of what order the symbols had been read by the reader. By this linking functionality, the QR Code will be able to be printed even if the printing space is not wide enough to have a single QR Code printed.

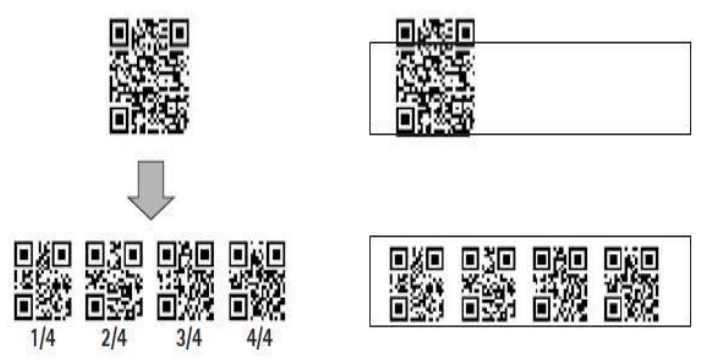

Figure 6: Linking the Symbols

\section{DESIGN OF QR CODE SCREENING SYSTEM}

\subsection{The framework of luggage inspection system}

Luggage inspection system, which is based on the QR code link structure, contains three main function module, which are generating QR code module, printing QR code module and decoding QR code module

\subsubsection{Generating $Q R$ code module}

This function module consists of the code program and generation of Boarding Pass with QR code printed on it shown in Figure 8, which include a concise user interface (UI) in Figure 7. The main input of UI include the passengers name, ID card number, the amount of luggage and related information to generate $\mathrm{QR}$ code sequence with link structure

\subsubsection{Printing $Q R$ code module}

QR code module support printer to print the QR code and Boarding Pass. Passengers only need to select anyone of the $\mathrm{QR}$ code symbols to print it on the boarding card, and the other QR code symbols are pasted on every luggage.

\subsubsection{Decoding QR code module}

This is the core function. All QR code symbol of the same sequence should be a unity and none of them can be lost because of the link structure characteristics. Decoding will success only when the barcode recognizer scans the QR code symbols all or it will fail. And it will make sure passengers get their own luggage. This is the vital characteristic of the luggage inspection system.

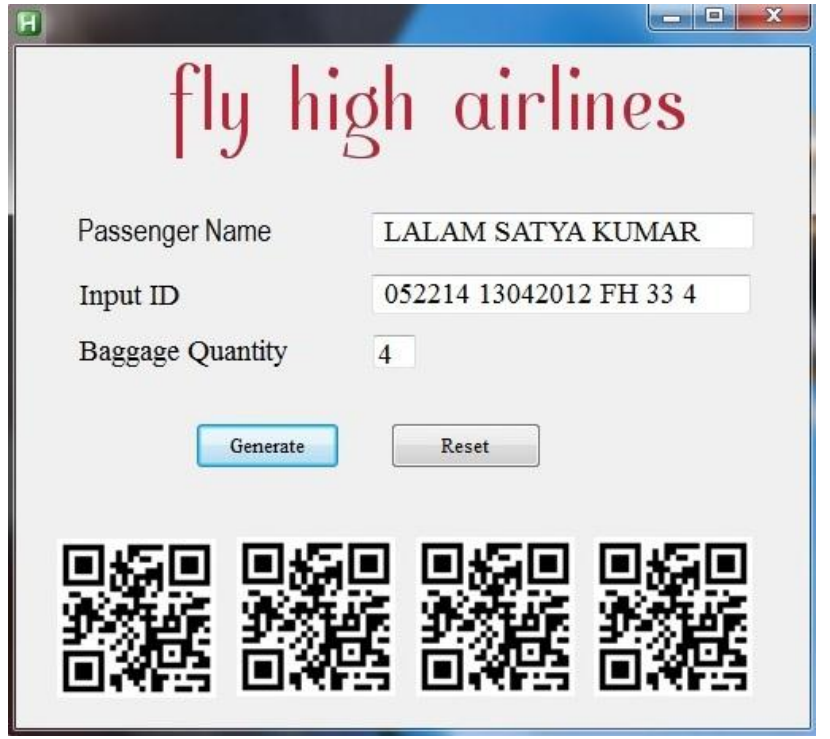

Figure 7: User Interface to Generate QR Code

\subsection{Process analysis of luggage inspection system}

The different from the traditional luggage inspection system is as follows. The first different from the traditional system is the barcode which is pasted on the baggage no longer is number code, but is the two dimensional bar code. The second different is when examining in the inspect port, the luggage is no longer checked by man-made operation, but checked by barcode recognizer. 


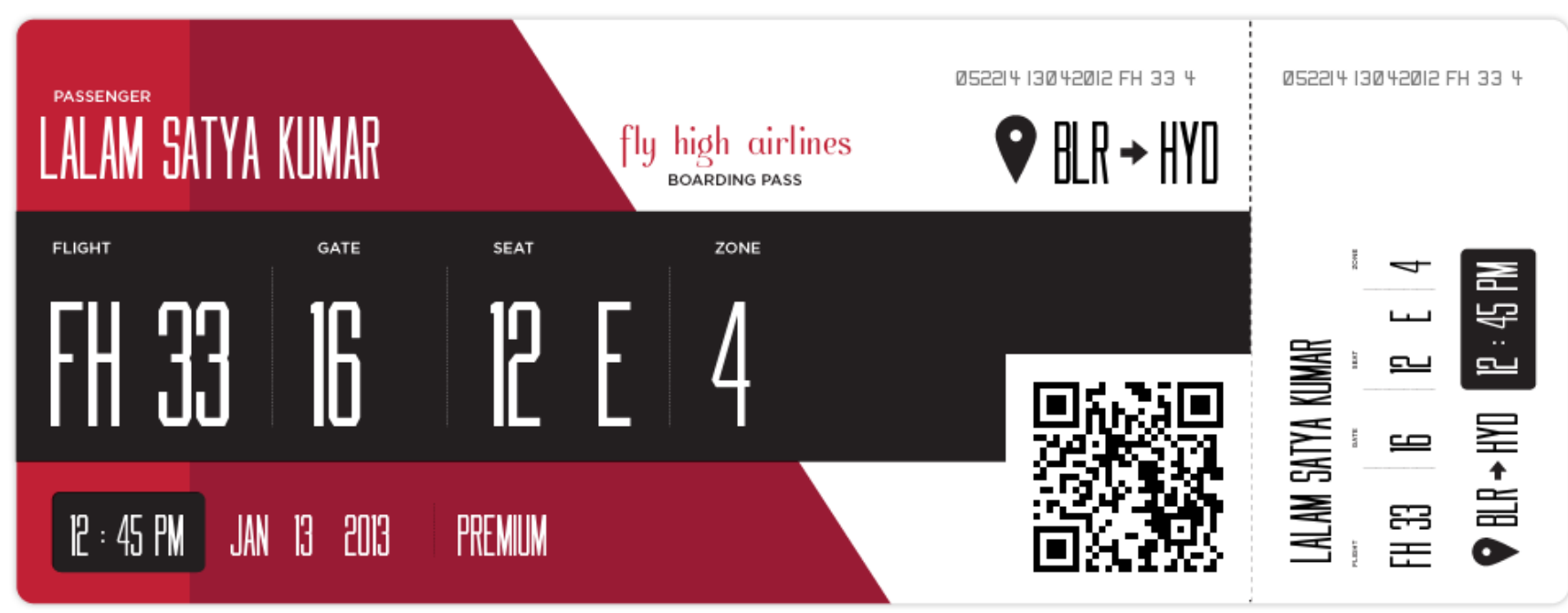

Figure 8: Generated Boarding Pass with QR Code

\section{ANALYSIS OF SYSTEM PERFORMANCE}

Compared with traditional manual luggage inspection methods, the new system uses a two dimension barcode technology, especially the link structure of the barcode. Meanwhile, the system doesn't need to refer to any database. Thus, the new system has its particular advantages as follows.

\subsection{Security analysis}

The system takes use of the link structure of OR code. That is to say, the only way to successfully decode is that the barcode recognizer scans the whole $\mathrm{QR}$ code sequence. As a result, passengers don't need to worry their luggage to be taken by others because of the similar look of luggage. Meanwhile, the system also checks whether some luggage are forgotten by passengers or not.

The system uses the barcode recognizer to scan, instead of the traditional manual inspection, so the error ratio by man-made decreases to a large extent. The operation of the whole system becomes safer and passengers become more enjoyable.

\subsection{Cost analysis}

Compare to the RFID technology, barcode is much cheaper than the RFID technology, the cost of barcode is only the cost of the paper and printing ink. In addition, this system doesn't need to establish a relevant database, so the system cost is reduced to a large extent.

\subsection{Efficiency analysis}

Because the system don't need database, it takes no more than 10 seconds in the process of generating and printing the QR code and no more than 3 seconds in processing of decoding symbol of QR code sequence. It takes the system less time to recognize the information and further make it more efficient. Compared with the traditional inspection methods, the efficiency of the system improves a lot. Moreover, from the angle of the barcode recognizer, QR code has itself characteristic to correct mistakes. Even when barcode was broken for more than 50 percent, it can read the information on the QR code symbol. So it has higher recognition rate compared to RFID technology.

\section{CONCLUSION}

This paper proposed a luggage inspection system based on the link structure of $\mathrm{QR}$ code, and give the design and implementation of the system in detail, which include the theory, framework and process of the system. Then, the performance of the system is compare and analysis with traditional method and RFID technology. Therefore, the system proposed will strengthen the luggage security and the inspection accuracy, which will enhance the speed and the efficiency of airport service, reduce the operation cost of airport, and improve satisfaction of passenger.

\section{REFERENCES}

[1] "QR Code features". Denso-Wave. 3 October 2011.

[2] "QR Code Standardization". QR Code.com. DensoWave. 23 April 2009.

[3] "Barcode Contents". zxing - A rough guide to standard encoding of information in barcodes.17 February 2009.

[4] "Consumer Alert: QR Code Safety". Better Business Bureau. 23 June 11.

[5] "Synchronization with Native Applications". NTT DoCoMo. Retrieved 17 February 2009.

[6] Aviation Enforcement and Proceedings, U.S. Department of Transportation. http://airconsumer.ost.dot.gov/reports/index.htm.

[7] Orli Sharaby (18 October 2010). "Form Meets Function: Extreme Makeover QR Code Edition".

[8] Denso (2009). Denso Wave Incorporated. June 21, 2010, from http://www.denso-wave.com/en/index.html.

[9] "Consumer Alert: QR Code Safety". Better Business Bureau. 23 June 11.

[10] "QR Code Standardization".QR Code.com. Denso-Wave. 